TecnoLógicas

ISSN-p 0123-7799

ISSN-e 2256-5337

Vol. 22, No. 44

Enero-abril de 2019

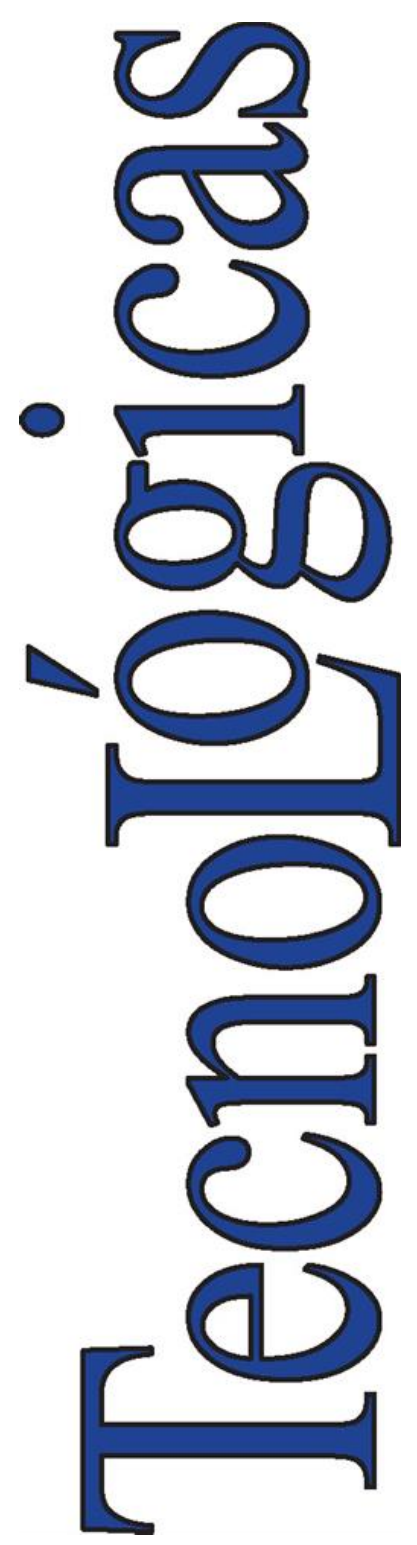

(C) Instituto Tecnológico Metropolitano Este trabajo está licenciado bajo una Licencia Internacional Creative Commons Atribución (CC BY-NC-SA)

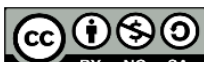

Artículo de Investigación/Research Article

\section{Modelo de asignación dinámica de lugares de almacenamiento en centros Cross Docking}

\section{Model of dynamic allocation of storage places in Cross Docking centers}

\author{
Sandra L. Rodríguez ${ }^{1}$, Viviana M. Díaz ${ }^{2}$, y \\ Juan P. Orejuela ${ }^{3}$
}

Recibido: 24 de abril de 2018

Aceptado: 22 de octubre de 2018

Cómo citar / How to cite

S. L. Rodríguez, V. M. Díaz, y J. P. Orejuela, Modelo de asignación dinámica de lugares de almacenamiento en centros Cross Docking. TecnoLógicas, vol. 22, no. 44, pp. 45-60, 2019.

https://doi.org/10.22430/22565337.743
1 Especialista en Higiene y Seguridad Industrial, Ingeniera Industrial, Escuela de Ingeniería Industrial, Universidad del Valle, Cali-Colombia, sandra.l.rodriguez@correounivalle.edu.co.

2 Especialista en Gestión de la Calidad Total y la Productividad, Escuela de Ingeniería Industrial, Universidad del Valle, Cali-Colombia, viviana.m.pulli@correounivalle.edu.co

3 MSc en Ingeniería Industrial, Escuela de Ingeniería Industrial, Universidad del Valle, Cali-Colombia, juan.orejuela@correounivalle.edu.co 


\section{Resumen}

Este trabajo describe una metodología para la asignación de lugares de almacenamiento temporal en centros tipo Cross Docking, para lo cual se desarrolla un modelo cuyo principal aporte es la asignación dinámica de productos entrantes a estos lugares de almacenamiento. El modelo minimiza los costos asociados al inventario final de los productos y los costos asociados a las distancias recorridas por unidad de carga. Se logra, además, una reducción del tiempo de permanencia y una aceleración del flujo de los productos desde la recepción hasta el despacho. Como principal conclusión, se identifica la importancia de las asignaciones puerta a puerta, en la reducción de los costos.

\section{Palabras clave}

Cross Docking, almacenamiento temporal, modelación matemática, asignación de productos a lugares de almacenamiento.

\section{Abstract}

This paper describes the methodology applicable to temporary storage in Cross Docking centers using a mathematical model. This model favors the dynamic allocation of storage places to incoming products in Cross Docking centers, reducing the costs associated with the final inventory of each of the products and the costs associated with the distances covered by each load unit. It also achieves a reduction in the time of permanence and an acceleration of the flow of products from reception to dispatch.

\section{Keywords}

Cross Docking, Temporary storage, mathematical modeling, product allocation to storage locations. 


\section{INTRODUCCIÓN}

Es común que las compañías busquen mejorar el desempeño en cada eslabón de su cadena de suministro, debido al ambiente globalizado y competitivo en el que se encuentran. En esta búsqueda, las compañías pueden llegar a desarrollar ventajas sobre sus competidores, enfocando sus esfuerzos principalmente en flexibilidad, la disminución de tiempo, costos y garantizando una mejor calidad de sus servicios [1].

Cada vez más se busca hacer las operaciones más eficientes, reducir costos, mediante la minimización del inventario en cada paso de la operación. Además los clientes están demandando mejores servicios, que se traducen en una entrega precisa y oportuna de los productos [1]. Proceso en el cual la gestión de almacenes juega un papel importante, ya que esta se ocupa de la organización de los productos que se tendrán en la bodega para lograr una gran utilización del espacio y facilitar el manejo eficiente del material [2].

La gestión de almacenes es un componente esencial en cualquier cadena de suministro. El uso eficiente de los recursos del almacén es necesario para aumentar la productividad y satisfacer las necesidades de demanda del cliente. El rendimiento de las operaciones de un almacén influye en el rendimiento de toda la cadena de suministro [3].

El Cross Docking se ha introducido como una estrategia de gestión de almacenamiento innovadora, que tiene una alta capacidad para manejar los costos de distribución mientras mantiene la satisfacción del cliente [4].

El objetivo principal de una terminal Cross Docking es permitir una consolidación de envíos de diferentes tamaños con el mismo destino a una carga completa de camiones, para que haya un ahorro en los costos de transporte. Esta ventaja hace que el Cross Docking sea una importante estrategia logística que recibe una mayor atención en los actuales días de globalización con un creciente volumen de productos transportado [5].

Los centros Cross Docking permiten acelerar el flujo de productos por medio de la recepción, movimiento y despacho casi inmediato a través de los centros de almacenamiento [6]. Lo que hace que estos se encuentren en una constante presión, ya que buscan reducir inventarios, los costos asociados a estos, los plazos de entrega, las distancias recorridas por cada uno de los productos y la permanencia de los productos en el centro.

En este sistema la eficiencia y efectividad se encuentran inversamente relacionadas con el tiempo que permanece un producto en el interior del centro [7], el tiempo para cada producto comienza a contar desde el momento que llega en un camión de entrada y termina cuando dicha unidad abandona la terminal en un camión de salida.

Según [8], en un centro de distribución el tiempo promedio que se consume en las actividades de recolección de órdenes corresponde aproximadamente en un $60 \%$ al desplazamiento, $25 \%$ extracción de los productos, $10 \%$ búsqueda y $5 \%$ asociado a otras tareas.

El Cross Docking, en comparación con el almacenamiento tradicional, elimina las funciones de almacenamiento $y$ preparación de pedidos de un almacén, mientras soporta sus funciones de recepción y envío. Por tanto, debe existir un equilibrio entre las cargas entrantes y salientes para que el Cross Docking tenga un buen rendimiento, ya que los productos se mantienen en un centro Cross Docking alrededor de 24 horas. Por lo tanto, los artículos que entran deberían ser demandados o pedidos por los clientes o puntos de destino rápidamente [4].

En tal sentido, la terminal CrossDocking es un nodo intermedio en una cadena de suministro, dedicada exclusivamente al transbordo de cargas de camiones. Cada vez que un camión 
entrante llega a la terminal Cross Docking, se asigna a una puerta del muelle, donde las cargas de entrada se descargan y escanean para determinar su destino. Las cargas se clasifican, se mueven a través del muelle y luego son cargadas en camiones de salida para una entrega inmediata en otro lugar en el sistema de distribución [5].

Las Investigaciones alrededor del Cross Docking se enfocan principalmente en aspectos estratégicos [9] y en aspectos tácticos [10]. En tal sentido, los problemas tratados en las investigaciones sobre este tema ordenados de estratégico a operativo son los siguientes [5]: Ubicación de la(s) terminal(es) Cross Docking. Diseño de la terminal. Asignación de destinos a las puertas de los muelles. Enrutamiento de vehículos. Programación de camiones. Programación de recursos dentro de la terminal. Embalaje o desembalaje de cargas en camiones. Algunas de estas investigaciones se presentan a continuación.

En [11] se evalúa la estrategia de envío directo de productos, con la estrategia de utilizar para el envío de productos una instalación intermedia de Cross Docking. El objetivo de este documento es evaluar las opciones de distribución y llevar a cabo un análisis comparativo exhaustivo con respecto a su costo-efectividad. El trabajo presenta como principal resultado las características del sistema que hace que una u otra estrategia sea mejor.

En [12] se propone un modelo cuyo objetivo es determinar la ubicación de los muelles Cross Docking, asignando proveedores a ellos y decisiones de enrutamiento, de modo que se minimizan el costo de ubicación y el costo total de envío en la red. Se utiliza un algoritmo meta heurístico llamado optimización basada en Biogeografía (BBO) para resolver el problema.

En [4] se presenta un modelo integrado para el diseño de sistemas Cross Docking en un entorno difuso; y en [13] se propone un modelo de programación entera mixta.
Ambos modelos tienen dos fases: un proceso de toma de decisiones estratégicas para seleccionar la ubicación de los muelles Cross Docking, y un proceso de toma de decisiones basado en operaciones para la programación de rutas de vehículos en múltiples centros Cross Docking.

Aunque el número de diferentes operaciones en un centro de Cross Docking es pequeño en comparación con un almacén de servicio completo, los problemas de planificación de estas son de una naturaleza compleja. Un problema de planificación de operaciones importante en los centros de Cross Docking se refiere a la asignación de la puerta del muelle. El cual consiste en asignar camiones entrantes y salientes a las puertas de carga y descarga, de modo que las operaciones dentro de las instalaciones se puedan realizar de la manera más eficiente posible [1].

En [14] se presenta un enfoque co- $^{-}$ evolutivo cooperativo para el enrutamiento y la programación de vehículos integrados utilizando el almacenamiento intermedio de Cross Docking. Se propone una formulación matemática bi-objetivo para el Cross Docking. Como el problema abordado es muy limitado, se desarrolla un enfoque de coevolución cooperativa, que consiste en híper heurística y heurística híbrida para lograr la mejora continua en objetivos alternativos.

En [15] se presenta integración entre la asignación de las puertas en centros Cross Docking y el problema de enrutamiento de vehículos. El objetivo es minimizar conjuntamente los costos totales de manipulación y transporte de materiales. Los autores presentan un algoritmo de generación de columnas basado en la formulación más prometedora y una heurística para obtenerlos límites inferior y superior para una solución óptima del problema.

En [16] se estudia el problema de programación de camiones en una terminal Cross Docking con múltiples puertas de recepción y envío. El objetivo es encontrar 
las mejores asignaciones de puertas, las secuencias de atraque tanto de camiones de entrada como de salida y también asignaciones de productos a camiones para minimizar el número ponderado de camiones de llegada tardía.

En [17] se aborda el concepto de JustIn-Time en asignación de puertas y la programación de camiones en un Centro Cross Docking de puertas y salidas múltiples, para ellos se desarrollan un modelo matemático de programación entera mixta que busca encontrar la programación de operaciones de transbordo para minimizar simultáneamente la precocidad total y la tardanza total de los camiones.

A partir de la revisión presentada, se puede decir que el Cross Docking es un tema interesante, tanto desde una perspectiva de investigación como desde un punto práctico. Sin embargo, se requieren más investigaciones alrededor de las decisiones de corto plazo corto plazo, dada su complejidad inherente [10]. En especiales en los tema la asignación de las puertas en los muelles y la ubicación de productos en el centro de almacenamiento[1].

En particular para el problema la ubicación de productos es importante tener presente que en la actualidad existen numerosas maneras de asignar los productos a los lugares de almacenamiento en bodegas tradicionales, entre ellas se encuentran la política de almacenamiento aleatorio, dedicado por familias y políticas que toman en cuenta el volumen de ventas de los productos. Sin embargo, estos métodos no son muy eficientes en centros Cross Docking debido a la diferencia que presentan estos comparados con los centros tradicionales [1].

Entre estas diferencias se tiene que en las bodegas tradicionales los productos son almacenados por largos periodos de tiempo, contrario a los centros Cross Docking, donde los productos llegan y son despachados en un tiempo mínimo aproximado de un día [1].

Otro factor diferenciador importante es que dado el poco tiempo que pasan los productos en la terminal y asimiento que se tiene espacio limitado que implique hacer un uso eficiente del espacio, el almacenamiento no debe ser dedicado si no dinámico.

Este problema de ubicación de productos en una terminal Cross Docking ha sido tratado en [1], donde se terminan mediante un algoritmo las ubicaciones de almacenamiento a corto plazo, de manera que las distancias totales de viaje en dentro de la instalación sean minimizada.

En la presente investigación se aborda el problema de la ubicación de productos y la asignación de las puestas de entrada y salida en un centro Cross Docking. Pero a diferencia del trabajo presentado en [1], en esta investigación se realiza un proceso de asignación de sitios de ubicación indexado en el tiempo, del tal modo que se obtiene una ubicación dinámica de los ítems en función del orden de llagada y salida de los camiones.

Para resolver el problema de asignación dinámica, se propone un modelo matemático de programación lineal entera, el cual se presenta a continuación.

\section{METODOLOGÍA}

La metodología propuesta se basa en el desarrollo de un modelo de Programación Lineal Entera Mixta para optimizar la asignación de lugares de almacenamiento a cada uno de los productos que ingresan a la terminal, minimizando las distancias totales de recorrido y los costos asociados a estas distancias.

\subsection{Supuestos del modelo}

Para la elaboración del modelo matemático, se tienen en cuenta los siguientes supuestos: 
-Existen dos tipos de lugares de almacenamiento, los tipos uno que corresponden a las localizaciones que se encuentran en el interior del centro Cross Docking (en estas los productos son almacenados temporalmente, por lo tanto, se considera el inventario) y los tipos dos que corresponden a las localizaciones ubicadas en las puertas de carga, es decir, cuando el producto es transportado directamente desde la puerta de descarga hasta la puerta de carga. En este tipo no hay consideración de inventario, ya que los camiones están ubicados en espera de ser despachados y no se permite obstaculizar el flujo de productos.

-A un lugar de almacenamiento solo se le puede asignar un tipo de producto en un instante de tiempo.

-Un producto puede ser asignado a varios lugares de almacenamiento.

-Las cantidades de producto $\mathbf{p}$ que entran y salen de la terminal son consideradas en unidades de carga.

\subsection{Notación y definición}

\section{Índices}

$\mathbf{p}, \mathbf{d}, \mathbf{a}, \mathbf{j}, \mathbf{c}, \mathbf{t}=$ Índices de: producto, puertas de descarga, lugares de almacenamiento, tipos de lugares de almacenamiento, puertas de carga, periodos de tiempo.

\section{Conjuntos}

$\mathbf{P R}=$ Productos $P R=\{1 \ldots n p\}$.

$\mathbf{P D}=$ Puertas de descarga $\mathrm{PD}=\{1 \ldots \mathrm{nd}\}$.

$\mathbf{L A}=$ Lugares de almacenamiento $L A=\{1 \ldots n a\}$.

$\mathbf{P C}=$ Puertas de carga $P C=\{1 \ldots n c\}$

$\mathbf{P T}=$ Periodos de tiempo $P T=\{1 \ldots t t\}$.

$\mathbf{T L}=$ Tipos de lugares de almacenamiento $T L=$ $\{1 \ldots t a\}$.

\section{Conjunto Inducido}

TipLoc [TL]= Localizaciones tipo $j$, el cual incluye las localizaciones tipo 1 y las localizaciones tipo 2 .

\section{Parámetros}

$\boldsymbol{n a}, \boldsymbol{n p}, \boldsymbol{n d}, \mathbf{n c}, \mathbf{t t}$, ta: Número de: localizaciones; productos; puertas de descarga; puertas de descarga; periodos de tiempo y tipos de localizaciones, respectivamente.

le $\boldsymbol{e}_{\boldsymbol{d} \boldsymbol{a}}=$ Distancia desde la puerta de descarga $d$ hasta el lugar de almacenamiento $a(\mathrm{~m})$.

$\boldsymbol{l} \boldsymbol{s}_{\boldsymbol{a}}=$ Distancia desde el lugar de almacenamiento $a$ hasta la puerta de carga $c(\mathrm{~m})$.

$\boldsymbol{k} \boldsymbol{t}=$ Capacidad total del centro de almacenamiento Cross Docking (unidades de carga).

$\boldsymbol{k}_{\boldsymbol{a}}=$ Capacidad del lugar de almacenamiento $a$. (unidades de carga).

$\boldsymbol{q} \boldsymbol{l}_{\boldsymbol{p d t}}=$ Cantidad de producto $p$ que llega a la puerta de descarga $d$ en el tiempo $t$.

$\boldsymbol{q} \boldsymbol{s}_{\boldsymbol{p} \boldsymbol{t}}=$ Cantidad del producto $\mathrm{p}$ que debe salir por la puerta de carga $c$ en el tiempo $t$.

$\boldsymbol{i p}_{\boldsymbol{p} \boldsymbol{a}}=$ Inventario inicial del producto $p$ en el lugar de almacenamiento $a$ en el tiempo $t$.

$\mathbf{i t}_{\mathbf{p}}=$ Inventario inicial del producto $p$ en toda la terminal.

$\mathbf{c l}_{\mathbf{p}}=$ Costo por metro de transportar la carga del producto $p$.

$\mathbf{c i}_{\mathbf{p a}}=$ Costo de almacenar el producto $p$ en el lugar de almacenamiento $a$.

\section{Variables de decisión}

$\mathbf{B L}_{\mathbf{p d a t}}=$ Variable binaria asociada a el producto $\boldsymbol{p}$ "1" si ingresa por la puerta de descarga $\boldsymbol{d}$ y se asigna al lugar de almacenamiento $\boldsymbol{a}$, en el tiempo $\boldsymbol{t}$, “0”de lo contrario.

BS $_{\text {pact }}=$ Variable binaria asociada a el producto $\boldsymbol{p}$ "1"si fue asignado al lugar de almacenamiento $\boldsymbol{a} y$ sale por la puerta de carga $\boldsymbol{c}$ en el tiempo $\boldsymbol{t}$, “0” de lo contrario.

$\mathbf{B I}_{\text {pat }}=$ Variable binaria asociada a el inventario final del producto $\boldsymbol{p}$ " 1 "si es mayor que cero en el lugar de almacenamiento $\boldsymbol{a}$, en el tiempo $\boldsymbol{t}$, “0” de lo contrario.

$\mathbf{Q E}_{\text {pdat }}=$ Cantidad enviada del producto $\boldsymbol{p}$ desde la puerta $\boldsymbol{d}$, hasta el lugar de almacenamiento $\boldsymbol{a}$, en el tiempo $\boldsymbol{t}$.

$\mathbf{Q S}_{\text {pact }}=$ Cantidad enviada del producto $\boldsymbol{p}$ desde el lugar de almacenamiento $\boldsymbol{a}$, hasta la puerta de carga $\boldsymbol{c}$, en el tiempo $\boldsymbol{t}$.

$\mathbf{F P}_{\text {pat }}=$ Inventario final del producto $\boldsymbol{p}$, en el lugar de almacenamiento $\boldsymbol{a}$, en el tiempo $\boldsymbol{t}$.

$\mathbf{F T}_{\mathbf{p t}}=$ Inventario final del producto $\boldsymbol{p}$, en toda la terminal, en el tiempo $\boldsymbol{t}$.

\subsection{Formulación matemática}

$\operatorname{Min}\left(\sum_{p \in P R} \sum_{d \in P D} \sum_{a \in L A} \sum_{t \in P T} c l_{p}\left(l e_{d a} \times B L_{p d a t}\right)+\sum_{p \in P R} \sum_{a \in L A} \sum_{c \in P C} \sum_{t \in P T} c l_{p}\left(l s_{a c} \times B S_{p a c t}\right)\right)+\left(\sum_{p \in P R} \sum_{a \in L A} \sum_{t \in P T} c i_{p a} \times F P_{p a t}\right)$ 


$$
\begin{aligned}
& \text { Restricciones } \\
& \sum_{p \in P R} B I_{p a t} \leq 1 \forall(a \in L A), \\
& \forall(t \in P T) \\
& q l_{p d t}=\sum_{a \in L A} Q E_{p d a t} \forall(p \in P R), \\
& \forall(d \in P D),(t \in P T) \\
& \sum_{p \in P R} \sum_{a \in L A} B L_{p d a t} \leq n a \\
& \forall(t \in P T) \forall(d \in P D) \\
& \sum_{p \in P R} \sum_{a \in L A} B I_{p a t} \leq n a \forall(t \in P T) \\
& Q E_{\text {pdat }} \leq B L_{\text {pdat }} \times k l_{a} \\
& \forall(p \in P R), \forall(d \in P D),(a \in L A),(t \in P T) \\
& Q S_{\text {pact }} \leq B S_{\text {pact }} \times k l_{a} \\
& \forall(p \in P R),(a \in L A), \forall(c \in P C),(t \in P T) \\
& \sum_{a \in L A} Q S_{p a c t}=q S_{p c t} \\
& \forall(p \in P R), \forall(c \in P C), \forall(t \in P T) \\
& \sum_{d \in P D} Q E_{\text {pdat }}=\sum_{c \in P C} Q S_{\text {pact }} \\
& \forall(p \in P R), \forall(a \in \text { TipLoc [2] }), \forall(t \in P T) \\
& F P_{\text {pat }}=0 \\
& \forall(p \in P R), \forall(a \in \text { TipLoc }[2]),(t \in P T) \\
& F P_{p a t}=i p_{p a}+\sum_{d \in P D} Q E_{p d a t}-\sum_{c \in P C} Q S_{p a c t} \text {, } \\
& \forall(p \in P R), \forall(a \in L A),(t=1) \\
& F P_{p a t}=F P_{p a(t-1)}+\sum_{d \in P D} Q E_{p d a t}-\sum_{c \in P C} Q S_{\text {pact }} \\
& \forall(p \in P R), \forall(a \in L A),(t>1) \\
& F T_{p t}=i t_{p}+\sum_{d \in P D} q l_{p d t}-\sum_{c \in P C} q s_{p c t}, \\
& \forall(p \in P R),(t=1) \\
& F T_{p t}=F T_{p(t-1)}+\sum_{d \in P D} q l_{p d t}-\sum_{c \in P C} q s_{p c t}
\end{aligned}
$$

$$
\begin{aligned}
& \forall(p \in P R),(t>1) \\
& F P_{p a t} \leq\left(B I_{p a t}\right) \times k l_{a} \\
& \forall(p \in P R), \forall(a \in L A),(t \in P T) \\
& B L_{p 1 d 1 a t}+B L_{p 2 d 2 a t} \leq 1 \\
& \forall(p \in P R), \forall(a \in L A),(t \in P T) \\
& B L_{p 1 d 1 a t}+B L_{p 2 d 2 a t} \leq 1 \\
& \forall\left(p_{1} \in P R\right), \forall\left(p_{2} \in P R\right), \forall(d 1 \in P D), \\
& \quad \forall(d 2 \in P D), \forall(a \in L A), \\
& B L_{p 2 d a t} \leq\left(1-B I_{p 1 a}(t-1)\right) M \\
& \forall\left(p_{1} \in P R\right), \forall\left(p_{2} \in P R\right), \forall(d \in P D), \\
& \quad \forall(a \in L A),(t \in P T), p_{1}<>p_{2} \\
& \sum_{d \in P D}^{B} L_{p d a t} \leq B I_{p a t} \times n d \\
& \forall(p \in P R), \forall(a \in L A), \forall(t \in P T), \\
& B L_{p d a t,} B S_{p a c t}, \mathrm{BI}_{p a t}=\text { Binaria } \\
& Q S_{p a c t,} \mathrm{FP}_{p a t}, \mathrm{FT}_{\mathrm{pt}}=\mathrm{Entera}
\end{aligned}
$$

La función objetivo (1), busca minimizar los costos de trasporte $\boldsymbol{c} \boldsymbol{l}_{\boldsymbol{p}}$ dentro del centro Cross Docking y los costos de mantener en inventario unidades de carga de los productos $\boldsymbol{c i}_{\boldsymbol{p} \boldsymbol{a}}$; (2) garantiza que un lugar de almacenamiento, en un momento de tiempo, solo puede recibir un tipo de producto; (3) garantiza que todo lo que llegue se localice; (4) garantiza que no se viole la capacidad en número de sitios de almacenamiento; (5) Garantiza que no se sobrepase la capacidad del centro de almacenamiento; ( 6 y $\quad 7$ ) garantizan la relación entre binarias y las cantidades de entrada y salida de producto, y el control de la capacidad en el lugar de almacenamiento; (8) garantiza que se cumpla el requerimiento en cada puerta de salida de cada producto; (9) garantiza el balance en las localizaciones tipo dos; (10) garantiza que el inventario final en las localizaciones tipo 2 sea cero; (11 y 12) definen el inventario final de producto en cada lugar de almacenamiento y en la 
terminal; (13) relaciona el inventario con la binaria de inventario; (14) garantiza que no se asigne a una localización, dos productos diferentes; (15 y 16) evitan que se asigna a una localización que tiene inventario de un producto otro producto diferente; (17 y 18) definen el tipo de variables.

\section{RESULTADOS Y DISCUSIÓN}

Esta sección presenta un ejemplo numérico para ilustrar las posibles aplicaciones del modelo propuesto para apoyar la asignación de productos a lugares de almacenamiento. Datos:

\subsection{La terminal}

En la Tabla 1 se presentan las características propias de la terminal Cross-Docking:

Tabla 1. Características de la terminal Cross Docking. Fuente: autores.

\begin{tabular}{|c|c|}
\hline $\begin{array}{l}\text { Puertas de } \\
\text { Descarga }\end{array}$ & $\begin{array}{l}\text { Se consideran dos }(2) \text { puertas de } \\
\text { descarga de los camiones. }\end{array}$ \\
\hline $\begin{array}{l}\text { Puertas de } \\
\text { Carga }\end{array}$ & $\begin{array}{l}\text { Dos }(2) \text { puertas de carga de } \\
\text { camiones de salida. }\end{array}$ \\
\hline $\begin{array}{l}\text { Número de } \\
\text { localizaciones }\end{array}$ & $\begin{array}{l}\text { Se consideran cinco }(5) \\
\text { localizaciones, tres (3) tipo } 1 \mathrm{y} \\
\text { dos }(2) \text { tipo } 2 .\end{array}$ \\
\hline $\begin{array}{l}\text { Capacidad } \\
\text { localizaciones } \\
\text { Tipo } 1 .\end{array}$ & 20 unidades de carga \\
\hline $\begin{array}{l}\text { Capacidad } \\
\text { localizaciones } \\
\text { Tipo } 2\end{array}$ & $\begin{array}{l}30 \text { unidades de carga } \\
\text { equivalentes a } 30 \text { pallets de } 1,0 \\
\text { x } 1,20 \text { mts. } \\
\text { Corresponden a capacidad de } \\
\text { carga de un camión de largo } \\
15.25 \text {, ancho } 2.45 \text {, y altura } 2.6 \\
\text { mts.) (Group, 2010) }\end{array}$ \\
\hline $\begin{array}{l}\text { Capacidad de } \\
\text { la terminal }\end{array}$ & 140 unidades de carga \\
\hline $\begin{array}{l}\text { Tipo de } \\
\text { productos } \\
\text { manejados }\end{array}$ & 3 tipos de productos.P1, P2, P3 \\
\hline $\begin{array}{l}\text { Periodos de } \\
\text { tiempo } \mathrm{t}\end{array}$ & $\mathrm{T} 1, \mathrm{~T} 2, \mathrm{~T} 3, \mathrm{~T} 4$ \\
\hline
\end{tabular}

\subsection{Las distancias}

En las Tablas 2 y 3 se detallan los parámetros de las distancias $l e_{d a} y l s_{c a}$, los cuales representan la distancia en metros desde la puerta de descarga hasta el lugar de almacenamiento y desde el lugar de almacenamiento hasta la puerta de carga respectivamente.

\subsection{Camiones de Ilegada y salida}

En las Tablas 4 y 5 se detallan las cantidades de cada tipo de producto que llegan en los camiones de entrada y se despachan en los camiones de salida. Tanto los camiones de entrada como los de salida tienen una capacidad de treinta (30) pallets y por lo general contienen más de 2 tipos de producto.

\subsection{Costos de transporte y almacenamiento temporal}

Con el fin de garantizar que los productos que entran a la terminal en los diferentes instantes de tiempo permanezcan el menor tiempo posible se penaliza el costo de mantener en inventario una unidad de carga el producto $p$ en el lugar de almacenamiento a.

Tabla 2. Distancias desde la puerta de descarga d hasta el lugar de almacenamiento a.

Fuente: autores.

\begin{tabular}{cccccc}
\hline \multicolumn{6}{c}{ Lugar de almacenamiento a } \\
\hline Puerta $d(m)$ & $\mathbf{1}$ & $\mathbf{2}$ & $\mathbf{3}$ & $\mathbf{4}$ & $\mathbf{5}$ \\
1 & 9,65 & 9,65 & 15,56 & 16,30 & 22,00 \\
2 & 15,56 & 9,65 & 9,65 & 22,00 & 16,30 \\
\hline
\end{tabular}

Tabla 3. Distancias desde el lugar de almacenamiento a hasta la puerta de carga $\mathrm{c}$. Fuente: autores.

\begin{tabular}{lcc}
\hline \multicolumn{3}{c}{ Puerta de carga $c$} \\
\hline Lugar $a(m)$ & $\mathbf{1}$ & $\mathbf{2}$ \\
1 & 9,65 & 14,06 \\
2 & 9,65 & 9,65 \\
3 & 15,56 & 9,65 \\
4 & 0,00 & 6,00 \\
5 & 6,00 & 0,00 \\
\hline
\end{tabular}


Tabla 4. Cantidad de producto p que llega por la puerta de descarga d. Fuente: autores.

\begin{tabular}{ccccccc}
\hline $\begin{array}{c}\text { Camió } \\
\mathrm{n}\end{array}$ & $\begin{array}{c}\text { Llegada } \\
(\mathrm{t})\end{array}$ & $\begin{array}{c}\text { Puerta de } \\
\text { descarga (d) }\end{array}$ & $\begin{array}{c}\text { Prod. 1 } \\
\text { (Und.) }\end{array}$ & $\begin{array}{c}\text { Prod. 2 } \\
\text { (Und.) }\end{array}$ & $\begin{array}{c}\text { Prod. 3 } \\
\text { (Und.) }\end{array}$ & $\begin{array}{c}\text { Carga Camión de } \\
\text { llegada }\end{array}$ \\
\hline A & 1 & 1 & 11 & 15 & 0 & 26 \\
B & 1 & 2 & 0 & 5 & 25 & 30 \\
C & 2 & 1 & 18 & 8 & 4 & 30 \\
D & 2 & 2 & 24 & 0 & 6 & 30 \\
E & 3 & 2 & 27 & 0 & 3 & 30 \\
F & 4 & 1 & 14 & 0 & 16 & 30 \\
G & 4 & 2 & 0 & 25 & 5 & 30 \\
\hline
\end{tabular}

Tabla 5. Cantidad de producto p que sale por la puerta de carga c. Fuente: autores.

\begin{tabular}{ccccccc}
\hline $\begin{array}{c}\text { Camió } \\
\mathrm{n}\end{array}$ & $\begin{array}{c}\text { Salida } \\
(\mathrm{t})\end{array}$ & $\begin{array}{c}\text { Puerta de } \\
\text { carga (d) }\end{array}$ & $\begin{array}{c}\text { Prod. 1 } \\
\text { (Und.) }\end{array}$ & $\begin{array}{c}\text { Prod. 2 } \\
\text { (Und.) }\end{array}$ & $\begin{array}{c}\text { Prod. 3 } \\
\text { Und.) }\end{array}$ & $\begin{array}{c}\text { Carga Camión de } \\
\text { llegada }\end{array}$ \\
\hline A & 1 & 1 & 0 & 9 & 20 & 29 \\
B & 2 & 1 & 4 & 9 & 8 & 21 \\
C & 2 & 2 & 0 & 10 & 7 & 17 \\
D & 3 & 2 & 27 & 0 & 3 & 30 \\
E & 4 & 1 & 26 & 0 & 0 & 26 \\
F & 4 & 2 & 28 & 0 & 0 & 28 \\
G & 4 & 2 & 0 & 25 & 5 & 30 \\
\hline
\end{tabular}

Tabla 6. Costos de almacenar el producto $p$ en el lugar de almacenamiento a (\$/unidad de carga). Fuente: autores.

\begin{tabular}{cccccc}
\hline \multicolumn{3}{c}{ Lugar de almacenamiento $\boldsymbol{a}$} \\
\hline Producto $\boldsymbol{p}$ & \multicolumn{2}{c}{ Tipo $\mathbf{1}$} & \multicolumn{3}{c}{ Tipo $\mathbf{2}$} \\
& 1 & 2 & 3 & 4 & 5 \\
$\mathbf{1}$ & 190 & 190 & 190 & 15 & 15 \\
$\mathbf{2}$ & 200 & 200 & 200 & 20 & 20 \\
$\mathbf{3}$ & 120 & 120 & 120 & 30 & 30 \\
\hline
\end{tabular}

Tabla 7. Costo de transportar una unidad de carga por metro de recorrido para cada producto $\mathrm{p}$ (\$/unidad de carga). Fuente: autores.

\begin{tabular}{cc}
\hline Producto $\boldsymbol{p}$ & $\begin{array}{c}\text { Costo de Transporte } \\
(\$)\end{array}$ \\
\hline $\mathbf{1}$ & 50 \\
$\mathbf{2}$ & 60 \\
$\mathbf{3}$ & 70 \\
\hline
\end{tabular}

Es de aclarar que para los lugares de almacenamiento tipo 2 , el costo es menor comparado con el costo de los lugares de almacenamiento tipo 1. En las Tablas 6 y 7 se presentan los costos de almacenar y transportar respectivamente.

\section{ANÁLISIS DE LOS RESULTADOS}

\subsection{Implementación}

Para la implementación de la metodología planteada, se utilizó el lenguaje de programación AMPL, en donde se cargaron los tres archivos, el primero denominado Modelo_asignación.txt en donde se describe la formulación matemática; el segundo de nombre Datos_asignación.txt en el cual se declaran los conjuntos simples, inducidos y los valores de los parámetros establecidos en el caso de estudio; por último, el archivo denominado Comando_Asignación.txt en el cual se declaran las variables que debe ejecutar dicho modelo. (Ver Anexo 1)

En la Tabla 8 se muestra la asignación de lugares de almacenamiento a unidades de carga de productos arrojada por el lenguaje de programación AMPL a través del solver $C B C$ utilizado para la programación lineal 
entera mixta, con el cual se obtuvo un costo de $\$ 47375.5$.

Del valor total de la función objetivo $\$ 20.235,5$ corresponden a los costos de trasportar y $\$ 27.140$ corresponden a los costos de mantener inventario.

El problema tiene 463 variables: 289 variables binarias y 174 variables enteras. Para su solución se realizaron 8586 iteraciones, 80 nodos, y su tiempo computacional fue de3.68244 segundos.

En la Tabla 8 se pueden ratificar los supuestos descritos anterior mente, entre ellos el que a un lugar de almacenamiento sólo se le puede asignar un tipo de producto en un instante de tiempo, pero un producto sí puede ser asignado a varios lugares de almacenamiento debido a las limitaciones de capacidad de cada uno de los lugares, cumpliendo con la función objetivo.

También se puede evidenciar la flexibilidad que tiene el modelo en cuanto a la entrada de productos por diferentes puertas en un mismo instante de tiempo tal como se muestran en el tercer y cuarto orden de asignación, en donde el producto 1 ingresa por la puerta 1 y por la puerta 2 en el instante de tiempo 2, sin embargo, éste se asigna al mismo lugar de almacenamiento, puesto que este cuenta con capacidad.

En la Tabla 9 se representan las cantidades de cada uno de los productos que fueron transportados desde las puertas de descarga hasta los lugares de almacenamiento (según la asignación de la binaria $\mathrm{BL}_{\text {pdat }}$ ) en cada uno de los instantes de tiempo, teniendo en cuenta las limitaciones de capacidades tanto de los lugares de almacenamiento tipo 1 como de los tipo 2, como modo ilustrativo se puede evidenciar que en la quinta asignación se transportaron 20 unidades de carga del producto 1 al lugar de almacenamiento 3, las cuales corresponden a la capacidad máxima de ese lugar ya que este pertenece a los tipo 1. Por otro lado, en la sexta asignación se transportaron 27 unidades de carga del producto 1 al lugar de almacenamiento 4 , el cual pertenece a los lugares de almacenamiento tipo 2 , además de ello el modelo está cumpliendo con la restricción que declara que si la cantidad que entra del producto $\boldsymbol{p}$, por la puerta $\boldsymbol{d}$ en el tiempo $\boldsymbol{t}$ igual a la cantidad que sale del producto $\boldsymbol{p}$ por la puerta $\boldsymbol{c}$ en el tiempo $\boldsymbol{t}$, asigne dicho producto a una de las localizaciones tipo 2 .

Tabla 8. Resultados de la variable de asignación. Fuente: autores.

\begin{tabular}{cccccc}
\hline $\begin{array}{c}\text { Orden de la } \\
\text { Asignación }\end{array}$ & $\begin{array}{c}\text { Produc } \\
\text { to } p\end{array}$ & $\begin{array}{c}\text { Puerta de } \\
\text { descarga } d\end{array}$ & $\begin{array}{c}\text { Lugar de almacenamiento } \\
\text { (asignado) }\end{array}$ & $t$ & $\begin{array}{c}\text { Valor de la binaria de } \\
\text { asignación }\end{array}$ \\
\hline 1 & 1 & 1 & 1 & 1 & 1.0 \\
2 & 1 & 1 & 1 & 4 & 1.0 \\
3 & 1 & 1 & 2 & 2 & 1.0 \\
4 & 1 & 2 & 2 & 2 & 1.0 \\
5 & 1 & 2 & 3 & 2 & 1.0 \\
6 & 1 & 2 & 4 & 3 & 1.0 \\
7 & 2 & 1 & 2 & 1 & 1.0 \\
8 & 2 & 1 & 4 & 2 & 1.0 \\
9 & 2 & 2 & 2 & 1 & 1.0 \\
10 & 2 & 2 & 5 & 4 & 1.0 \\
11 & 3 & 1 & 2 & 4 & 1.0 \\
12 & 3 & 1 & 5 & 2 & 1.0 \\
13 & 3 & 2 & 2 & 4 & 1.0 \\
14 & 3 & 2 & 3 & 1 & 1.0 \\
15 & 3 & 2 & 4 & 1 & 1.0 \\
16 & 3 & 2 & 5 & 2 & \\
17 & 3 & 2 & 5 & 3 & \\
\hline
\end{tabular}


Tabla 9. Cantidad transportada del producto $p$ hacia los lugares de almacenamiento $a$. Fuente: autores.

\begin{tabular}{cccccc}
\hline $\begin{array}{c}\text { Orden de la } \\
\text { Asignación }\end{array}$ & $\begin{array}{c}\text { Produc } \\
\text { to } p\end{array}$ & $\begin{array}{c}\text { Puerta de } \\
\text { descarga } d\end{array}$ & $\begin{array}{c}\text { Lugar de } \\
\text { almacenamiento } a\end{array}$ & $\begin{array}{c}\text { Instante de } \\
\text { tiempo } t\end{array}$ & $\begin{array}{c}\text { Cantidad enviada al } \\
\text { lugar de } \\
\text { almacenamiento a }\end{array}$ \\
\hline 1 & 1 & 1 & 1 & 1 & 11.0 \\
2 & 1 & 1 & 1 & 4 & 14.0 \\
3 & 1 & 1 & 2 & 2 & 18.0 \\
4 & 1 & 2 & 2 & 2 & 4.0 \\
5 & 1 & 2 & 3 & 2 & 20.0 \\
6 & 1 & 2 & 4 & 3 & 27.0 \\
7 & 2 & 1 & 2 & 1 & 15.0 \\
8 & 2 & 1 & 4 & 2 & 8.0 \\
9 & 2 & 2 & 2 & 1 & 5.0 \\
10 & 2 & 2 & 5 & 4 & 25.0 \\
11 & 3 & 1 & 2 & 4 & 16.0 \\
12 & 3 & 1 & 5 & 2 & 4.0 \\
13 & 3 & 2 & 2 & 4 & 5.0 \\
14 & 3 & 2 & 3 & 1 & 5.0 \\
15 & 3 & 2 & 4 & 1 & 20.0 \\
16 & 3 & 2 & 5 & 2 & 6.0 \\
17 & 3 & 2 & 5 & 3 & 3.0 \\
\hline
\end{tabular}

Tabla 10. Cantidad transportada del producto $p$ desde el lugar de almacenamiento $a$ hasta la puerta de carga $c$.

\begin{tabular}{|c|c|c|c|c|c|}
\hline $\begin{array}{l}\text { Orden de la } \\
\text { Asignación }\end{array}$ & $\begin{array}{c}\text { Producto } \\
\boldsymbol{p}\end{array}$ & $\begin{array}{c}\text { Lugar de } \\
\text { almacenamiento } \boldsymbol{a}\end{array}$ & $\begin{array}{l}\text { Puerta de } \\
\text { carga } \boldsymbol{c}\end{array}$ & $\begin{array}{c}\text { Instante de } \\
\text { tiempo } t\end{array}$ & $\begin{array}{c}\text { Cantidad enviada a la } \\
\text { puerta de carga c }\end{array}$ \\
\hline 1 & 1 & 1 & 1 & 4 & 20.0 \\
\hline 2 & 1 & 2 & 1 & 2 & 4.0 \\
\hline 3 & 1 & 2 & 1 & 4 & 6.0 \\
\hline 4 & 1 & 2 & 2 & 4 & 12.0 \\
\hline 5 & 1 & 3 & 2 & 4 & 16.0 \\
\hline 6 & 1 & 4 & 2 & 3 & 27.0 \\
\hline 7 & 2 & 2 & 1 & 1 & 9.0 \\
\hline 8 & 2 & 2 & 1 & 2 & 1.0 \\
\hline 9 & 2 & 2 & 2 & 2 & 10.0 \\
\hline 10 & 2 & 4 & 1 & 2 & 8.0 \\
\hline 11 & 2 & 5 & 2 & 4 & 25.0 \\
\hline 12 & 3 & 2 & 2 & 4 & 5.0 \\
\hline 13 & 3 & 3 & 2 & 2 & 5.0 \\
\hline 14 & 3 & 4 & 1 & 1 & 20.0 \\
\hline 15 & 3 & 5 & 1 & 2 & 8.0 \\
\hline 16 & 3 & 5 & 2 & 2 & 2.0 \\
\hline 17 & 3 & 5 & 2 & 3 & 3.0 \\
\hline
\end{tabular}

De la misma manera en la Tabla 10 se registran las cantidades de cada uno de los productos $\boldsymbol{p}$ que fueron transportados a las puertas de carga según los requerimientos de salida en los diferentes instantes de tiempo, ratificando una vez más el cumplimiento de la capacidad de cada uno de los lugares de almacenamiento y la equivalencia entre las unidades de carga que ingresan a la terminal y las que salen.

\subsection{Implementación análisis de sensibilidad}

El análisis de sensibilidad se relaciona con la cuantificación de los efectos que se generan en la solución óptima, al realizar cambios en los parámetros del modelo matemático.

Para el análisis de sensibilidad de las distintas instancias planteadas se decidió variar los parámetros de costos de transporte y los costos mantener 
inventario los cuales conforman la función objetivo, de la siguiente forma:

\section{Variación de costos de transporte}

El parámetro $c l_{p}$ (costo de trasporte del producto $\boldsymbol{p}$ ) se planteó en el caso de estudio inicialmente con un valor de 50, 60 y 70 pesos por cada uno de los productos respectivamente.

Para el análisis de sensibilidad del modelo propuesto frente a este parámetro, se decidió fijar el parámetro $c i_{p a}$ (costo de mantener en inventario el producto $\boldsymbol{p}$ en el lugar de almacenamiento a) los cuales tienen el valor de 190, 200 y 120 pesos para cada uno de los productos respectivamente en las localizaciones tipo 1 y 15,20 y 30 pesos para las localizaciones tipo 2. La variación del costo de transporte se realizó en porcentajes mayores al costo planteado en el caso de estudio tal como se muestra en la Tabla 11.

Tabla 11. Variación positiva de los costos de transporte del producto p. Fuente: autores.

\begin{tabular}{ccccccc}
\hline \multicolumn{6}{c}{ Variación Costo de Transporte } \\
\hline Producto & 0 & 100 & 200 & 300 & 400 & 500 \\
& $\%$ & $\%$ & $\%$ & $\%$ & $\%$ & $\%$ \\
1 & 50 & 100 & 150 & 200 & 250 & 300 \\
2 & 60 & 120 & 180 & 240 & 300 & 360 \\
3 & 70 & 140 & 210 & 280 & 350 & 420 \\
\hline
\end{tabular}

Al realizar la variación del parámetro de costo de transporte, se cargaron los archivos correspondientes en el lenguaje de programación AMPL, y se consolidaron los resultados obtenidos para la función objetivo, tal como se muestra en la Tabla 12:

De los anteriores resultados, se puede identificar que a medida que el costo de trasporte se incrementa, se presenta un aumento en el valor de la función objetivo en una proporción menor al aumento del costo, ya que si se realiza un aumento del costo de $500 \%$ el porcentaje de aumento de la función objetivo es de $214 \%$ con un costo de $\$ 148.553,00$ (ver Fig. 1).

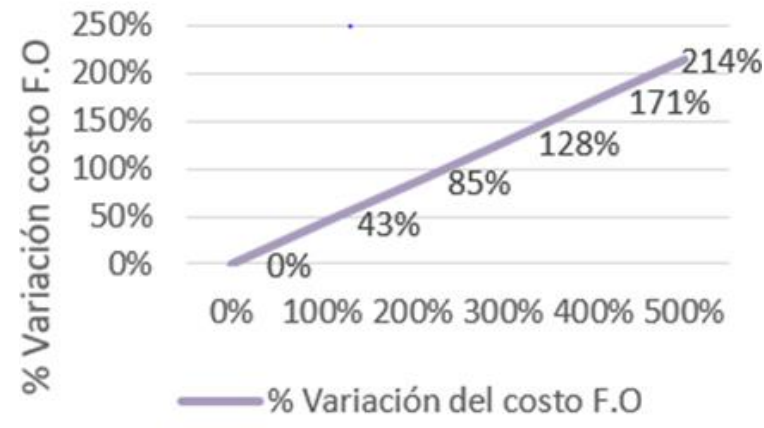

\% Variación del costo de Trasportar

Fig. 1. Variación costo de trasporte vs variación Costo F.O. Fuente: autores.

Tabla 12. Resultados de la variación del costo de transporte. Fuente: autores.

\begin{tabular}{|c|c|c|c|}
\hline $\begin{array}{l}\text { No. De } \\
\text { prueba }\end{array}$ & $\begin{array}{l}\text { Costo } \\
\text { F.O }\end{array}$ & $\begin{array}{c}\text { Variación } \\
\text { Costo de } \\
\text { Transportar }\end{array}$ & $\begin{array}{l}\text { Variación } \\
\text { costo F.O }\end{array}$ \\
\hline 0 & $\begin{array}{c}\$ \\
47.375,50\end{array}$ & $0 \%$ & $0 \%$ \\
\hline 1 & $\begin{array}{c}\$ \\
67.611,00\end{array}$ & $100 \%$ & $43 \%$ \\
\hline 2 & $\begin{array}{c}\$ \\
87.846,50\end{array}$ & $200 \%$ & $85 \%$ \\
\hline 3 & $\begin{array}{c}\$ \\
108.082,00\end{array}$ & $300 \%$ & $128 \%$ \\
\hline 4 & $\begin{array}{c}\$ \\
128.317,50\end{array}$ & $400 \%$ & $171 \%$ \\
\hline 5 & $\begin{array}{c}\$ \\
148.553,00\end{array}$ & $500 \%$ & $214 \%$ \\
\hline
\end{tabular}

\section{Variación del costo de mantener inventario}

Consiste en variar el parámetro $\mathrm{ci}_{\mathrm{pa}}$ (costo de mantener en inventario una unidad de carga del producto $p$ en el lugar de almacenamiento a) de manera positiva, mantenimiento fijo el parámetro $\mathrm{cl}_{\mathrm{p}}$ (costo de transporte de una unidad de carga del producto $\mathrm{p}$, por metro recorrido), para esta instancia se debe tener en cuenta que los costos de mantener inventario son diferentes por cada tipo de localización ya que el costo de mantener inventario en las tipo 2 es mucho menor que el costo de mantener inventario en las tipo 1.

En las Tablas 13 y 14 se muestran las correspondientes variaciones que se realizaron: 
Tabla 13. Variación positiva del costo de mantener inventario para las localizaciones tipo 1. Fuente: autores.

\begin{tabular}{ccccccc}
\hline \multicolumn{7}{c}{ Variación Costo de mantener en inventario una } \\
unidad de carga \\
\hline Prod. $p$ & $0 \%$ & 100 & $200 \%$ & 300 & 400 & 500 \\
& & $\%$ & & $\%$ & $\%$ & $\%$ \\
$\mathbf{1}$ & 15 & 30 & 45 & 60 & 75 & 90 \\
$\mathbf{2}$ & 20 & 40 & 60 & 80 & 100 & 120 \\
$\mathbf{3}$ & 30 & 60 & 90 & 120 & 150 & 180 \\
\hline
\end{tabular}

$\mathrm{Al}$ realizar la variación del parámetro de costo de mantenimiento de inventario, se cargaron los archivos correspondientes en el lenguaje de programación AMPL, y se consolidaron los resultados obtenidos para la función objetivo, tal como se muestra en la Tabla 15.

Tabla 14. Variación positiva del costo de mantener inventario para las localizaciones tipo 2 .

Fuente: autores.

\begin{tabular}{ccccccc}
\hline \multicolumn{7}{c}{ Variación Costo de mantener en inventario } \\
una unidad de carga \\
\hline $\begin{array}{c}\text { Prod. } \\
\text { p }\end{array}$ & $0 \%$ & $100 \%$ & $200 \%$ & $300 \%$ & $400 \%$ & $500 \%$ \\
\hline 1 & 190 & 380 & 570 & 760 & 950 & 1140 \\
2 & 200 & 400 & 600 & 800 & 1000 & 1200 \\
3 & 120 & 240 & 360 & 480 & 600 & 720 \\
\hline
\end{tabular}

Tabla 15. Resultados de la variación del costo de mantener el inventario. Fuente: autores.

\begin{tabular}{cccc}
\hline $\begin{array}{c}\text { No. De } \\
\text { prueba }\end{array}$ & Costo F.O & $\begin{array}{c}\text { Variación } \\
\text { Costo de } \\
\text { mantener } \\
\text { inventario }\end{array}$ & $\begin{array}{c}\text { Variación } \\
\text { costo F.O }\end{array}$ \\
\hline 0 & $\$ 47.375,50$ & $0 \%$ & $0 \%$ \\
1 & $\$ 74.515,50$ & $100 \%$ & $57 \%$ \\
2 & $\$ 101.655,50$ & $200 \%$ & $115 \%$ \\
3 & $\$ 129.209,20$ & $300 \%$ & $173 \%$ \\
4 & $\$ 155.935,50$ & $400 \%$ & $229 \%$ \\
5 & $\$ 183.075,50$ & $500 \%$ & $286 \%$ \\
\hline
\end{tabular}

De la Tabla 15 se puede inferir que la variación del costo de la función objetivo se realiza en menor proporción que la variación del costo de mantener inventario, ya que, si se aumenta un $500 \%$ el valor del costo de mantener inventario, la función objetivo presenta un aumento del $286 \%$ con un costo de $\$ 183.075,50$.

En la Fig. 2, se puede observar las variaciones del costo de mantener inventario versus la variación que representa el costo de la función objetivo.

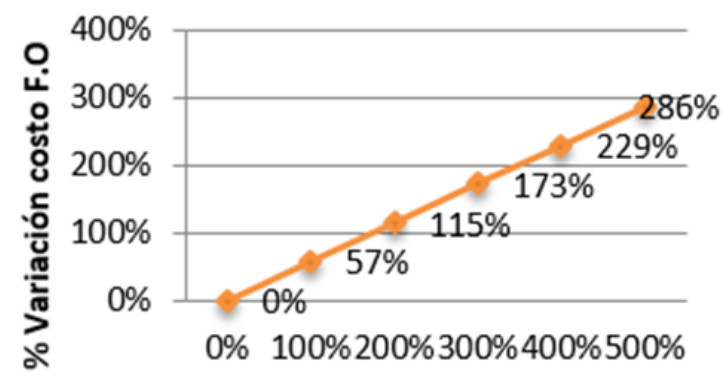

\% Variación Costo de mantener inventario

Fig. 2. Variación positiva del costo de mantener inventario vs la variación del costo de la F.O.

Fuente: autores.

\section{Variación del número de productos $y$ del número de lugares de almacenamiento}

Para esta instancia se aumentó tanto el número de productos como el número de lugares de almacenamiento, ya que la relación que existe entre estos parámetros es directamente proporcional debido a que un producto $\boldsymbol{p}$ solo puede ser almacenado en un lugar de almacenamiento $\boldsymbol{a}$.

En las Tablas 16, 17 y 18 se muestra un resumen de los resultados de la instancia variando el número de localizaciones y al mismo tiempo el número de productos, en la Fig. 3, se puede observar que a medida que el número de productos aumenta, para todos los casos el número de nodos, de interacciones $\mathrm{y}$ el tiempo computacional son mayores.

Tabla 16. Resultados del modelo con ocho (8) localizaciones. Fuente: autores.

\begin{tabular}{ccccc}
\hline $\begin{array}{c}\mathrm{N}^{\circ} \text { de } \\
\text { Prod. }\end{array}$ & $\begin{array}{c}\text { Valor } \\
\text { F.O }\end{array}$ & $\begin{array}{c}\text { Tiempo } \\
\text { comp (seg) }\end{array}$ & $\begin{array}{c}\mathrm{N}^{\mathrm{o}} \mathrm{de} \\
\text { nodos }\end{array}$ & $\begin{array}{c}\mathrm{N}^{\mathrm{o}} \mathrm{de} \\
\text { iter. }\end{array}$ \\
\hline $\mathbf{1}$ & 10715 & 0,074989 & 0 & 0 \\
$\mathbf{2}$ & 22731,6 & 4,706290 & 14 & 4406 \\
$\mathbf{3}$ & 36577,3 & 43,74340 & 462 & 54627 \\
$\mathbf{4}$ & 55790,2 & 112,1740 & 797 & 157288 \\
$\mathbf{5}$ & 71320,9 & 7635,32 & 42236 & 13834184 \\
\hline
\end{tabular}

El valor de la función objetivo para uno y dos productos no sufre ninguna variación 
en los tres escenarios, esto se debe a que el modelo está transportando y almacenando uno o dos productos y aunque se tengan muchas localizaciones disponibles, el modelo escoge la mejor, ya que no tiene restricciones para almacenar. Sin embargo, para tres, cuatro o cinco productos, el valor de la función objetivo empieza con algunas variaciones mínimas, esto se debe al aumento del número de productos, ya que las mejores localizaciones están ocupadas y por lo tanto el modelo debe elegir otra $\mathrm{y}$ esto ocasiona que el valor aumente para algunos casos o para los tres escenarios.

Tabla 17. Resultados del modelo con nueve (9) localizaciones. Fuente: autores.

\begin{tabular}{|c|c|c|c|c|}
\hline $\begin{array}{l}\mathrm{N}^{\circ} \text { de } \\
\text { Prod. }\end{array}$ & $\begin{array}{l}\text { Valor } \\
\text { F.O }\end{array}$ & $\begin{array}{c}\text { Tiempo } \\
\text { computaci } \\
\text { onal (seg) }\end{array}$ & $\begin{array}{l}\mathrm{N}^{\mathrm{o}} \mathrm{de} \\
\text { nodos }\end{array}$ & $\begin{array}{c}\mathrm{N}^{\mathrm{o}} \mathrm{de} \\
\text { iteraciones }\end{array}$ \\
\hline 1 & 10715 & 0,099985 & 0 & 0 \\
\hline 2 & 22731,6 & 4,706290 & 16 & 3455 \\
\hline 3 & 36222,1 & 42,76750 & 662 & 79147 \\
\hline 4 & 55240,6 & 183,7840 & 1065 & 251466 \\
\hline
\end{tabular}

Tabla 18. Resultados del modelo con diez (10) localizaciones. Fuente: autores.

\begin{tabular}{|c|c|c|c|c|}
\hline $\begin{array}{l}\text { No de } \\
\text { Prod. }\end{array}$ & F.O & $\begin{array}{c}\text { Tiempo } \\
\text { computaci } \\
\text { onal (seg) }\end{array}$ & $\begin{array}{l}\mathrm{N}^{\mathrm{o}} \mathrm{de} \\
\text { nodos }\end{array}$ & $\begin{array}{c}\mathrm{N}^{\mathrm{o}} \mathrm{de} \\
\text { iteraciones }\end{array}$ \\
\hline 1 & 10715 & 0,07099 & 0 & 0 \\
\hline 2 & 22731,6 & 6,360030 & 38 & 4893 \\
\hline 3 & 36222,1 & 30,94830 & 469 & 46927 \\
\hline 4 & 55051,9 & 69,62340 & 720 & 115898 \\
\hline 5 & 66073,4 & 483,486 & 2967 & 900221 \\
\hline
\end{tabular}

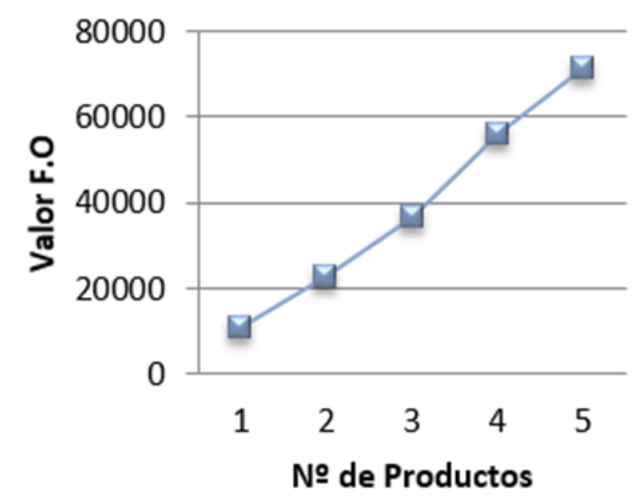

Fig. 3. Valor Función Objetivo vs Variación número de Productos. Fuente: autores.

También se puede observar que a medida que el número de productos aumenta el valor de la función objetivo como se observa en la Fig. 3.

\section{CONCLUSIONES}

Este modelo logra obtener una adecuada utilización del espacio, ya que permite ubicar unidades de carga de un mismo producto en lugares que ya han sido asignados con anterioridad, pero que tienen capacidad de almacenamiento disponible. Además, permite al administrador del centro conocer el inventario de los productos en cada uno de los lugares de almacenamiento en el periodo de tiempo que desee, liberando los lugares de almacenamiento que no cuenten con inventario final de producto para que estos sean asignados nuevamente, lo que se traduce en una utilización adecuada del espacio designado para almacenar.

El modelo de asignación que se presentó en esta investigación puede ser usado para diferentes casos de estudio en los cuales se puede variar el diseño del centro, el número de puertas de descarga (entrada), número de puertas de carga (salida), el número de productos y el número de lugares de almacenamiento temporales, teniendo en cuenta que entre mayor sea el tamaño del modelo mayor será el tiempo computacional requerido para su solución o podría ser imposible de resolverse.

Debido a que una de las principales limitantes para la optimización global de cualquier problema de asignación, es la complejidad computacional por el crecimiento exponencial del espacio de soluciones conforme crece el tamaño del problema para futuras investigaciones se pueden emplear técnicas heurísticas, con las cuales se puede dar solución a este inconveniente por medio de una búsqueda dirigida y eficiente en términos de tiempo computacional.

Como resultado final, obtuvo una correcta asignación de los lugares de 
almacenamiento temporales, es decir, el modelo asignó las unidades de carga unitaria de cada producto (desde la llegada de los productos a la puertas de descarga) a los lugares de almacenamiento disponibles que presentaron una distancia más corta de desplazamiento y según los requerimiento de unidades carga que se definieron para cada producto en las puertas de salida, se extrajeron los productos que estuvieran en el lugar de almacenamiento más cercano a estas, de manera que los costos de transporte fueron mínimos para así cumplir con el objetivo de minimizar el valor de la función objetivo.

La formulación matemática del modelo permite pasar directamente de puerta a puerta las unidades unitarias de los productos que requieren ser despachados en un mismo periodo de tiempo sin que se incurra en costo de almacenamiento, siendo esta una característica propia de una terminal Cross Docking - pura, y una muestra de flexibilidad del modelo presentado.

El empleo de este tipo de herramientas, es de gran ayuda para los tomadores de decisiones, ya que les permite proyectar la evaluación del impacto de sus decisiones con respecto a uno de los principales indicadores en los centros de distribución donde se maneja inventario, y más aún, cuando el centro de distribución es de tipo Cross Docking y su principal objetivo es reducir el tiempo de permanencia de los productos dentro de éstos.

\section{REFERENCIAS}

[1] I. F. A. Vis and K. J. Roodbergen, "Positioning of goods in a cross-docking environment," Comput. Ind. Eng., vol. 54, no. 3, pp. 677-689, Apr. 2008.

[2] S. Quintanilla, Á. Pérez, F. Ballestín, and P. Lino, "Heuristic algorithms for a storage location assignment problem in a chaotic warehouse," Eng. Optim., vol. 47, no. 10, pp. 1405-1422, Oct. 2015.

[3] M. Drakaki and P. Tzionas, "Modeling and performance evaluation of an agent-based warehouse dynamic resource allocation using Colored Petri Nets," Int. J. Comput. Integr. Manuf., vol. 29, no. 7, pp. 736-753, Jul. 2016.

[4] S. M. Mousavi, R. Tavakkoli-Moghaddam, and F. Jolai, "A possibilistic programming approach for the location problem of multiple cross-docks and vehicle routing scheduling under uncertainty," Eng. Optim., vol. 45, no. 10, pp. 1223-1249, Oct. 2013.

[5] N. Boysen and M. Fliedner, "Cross dock scheduling: Classification, literature review and research agenda," Omega, vol. 38, no. 6, pp. 413-422, Dec. 2010.

[6] M. R. Galbreth, J. A. Hill, and S. Handley, "An investigation of the value of cross-docking for supply chain management," J. Bus. Logist., vol. 29, no. 1, pp. 225-239, 2011.

[7] U. M. Apte and S. Viswanathan, "Effective Cross Docking for Improving Distribution Efficiencies," Int. J. Logist. Res. Appl., vol. 3, no. 3, pp. 291-302, Nov. 2000.

[8] C. J. Vidal, Planeación, Optimización y Administración de Cadenas de Abastecimiento, Primera Ed. Cali: Programa Editorial Universidad del Valle, 2010.

[9] N. Boysen, "Truck scheduling at zeroinventory cross docking terminals," Comput. Oper. Res., vol. 37, no. 1, pp. 32-41, Jan. 2010.

[10] D. Agustina, C. K. M. Lee, and R. Piplani, "A Review: Mathematical Modles for Cross Docking Planning," Int. J. Eng. Bus. Manag., vol. 2, no. 2, p. 13, Mar. 2010.

[11] A. I. Nikolopoulou, P. P. Repoussis, C. D. Tarantilis, and E. E. Zachariadis, "Moving products between location pairs: Crossdocking versus direct-shipping," Eur. J. Oper. Res., vol. 256, no. 3, pp. 803-819, Feb. 2017.

[12] A. Hasani Goodarzi and S. H. Zegordi, "A location-routing problem for cross-docking networks: A biogeography-based optimization algorithm," Comput. Ind. Eng., vol. 102, pp. 132-146, Dec. 2016.

[13] S. M. Mousavi and R. TavakkoliMoghaddam, "A hybrid simulated annealing algorithm for location and routing scheduling problems with cross-docking in the supply chain," J. Manuf. Syst., vol. 32, no. 2, pp. 335-347, Apr. 2013.

[14] P.-Y. Yin, S.-R. Lyu, and Y.-L. Chuang, "Cooperative coevolutionary approach for integrated vehicle routing and scheduling using cross-dock buffering," Eng. Appl. Artif. Intell., vol. 52, pp. 40-53, Jun. 2016.

[15] F. Enderer, C. Contardo, and I. Contreras, "Integrating dock-door assignment and vehicle routing with cross-docking," Comput. Oper. Res., vol. 88, pp. 30-43, Dec. 2017. 
[16] M. T. Assadi and M. Bagheri, "Scheduling trucks in a multiple-door Cross Docking system with unequal ready times," Eur. J. Ind. Eng., vol. 10, no. 1, p. 103, 2016.
[17] W. Wisittipanich and P. Hengmeechai, "A Multi-Objective Differential Evolution for JustIn-Time Door Assignment and Truck Scheduling in Multi-door Cross Docking Problems," Ind. Eng. Manag. Syst., vol. 14, no. 3, pp. 299-311, Sep. 2015.

[60] TecnoLógicas, ISSN-p 0123-7799 / ISSN-e 2256-5337, Vol. 22, No. 44, enero-abril de 2019, pp. 45-60 